\title{
THE
}

4-16-2003

\section{Quantum Size Effect in Conductivity of Multilayer Metal Films}

\author{
A. E. Meyerovich \\ University of Rhode Island, sfo101@uri.edu
}

I. V. Ponomarev

Follow this and additional works at: https://digitalcommons.uri.edu/phys_facpubs

Terms of Use

All rights reserved under copyright.

\section{Citation/Publisher Attribution}

Meyerovich, A. E., \& Ponomarev, I. V. (2003). Quantum Size Effect in Conductivity of Multilayer Metal Films. Phys. Rev. B, 67, 165411. doi: 10.1103/PhysRevB.67.165411

Available at: http://dx.doi.org/10.1103/PhysRevB.67.165411

This Article is brought to you for free and open access by the Physics at DigitalCommons@URI. It has been accepted for inclusion in Physics Faculty Publications by an authorized administrator of DigitalCommons@URI. For more information, please contact digitalcommons-group@uri.edu. 


\title{
Quantum size effect in conductivity of multilayer metal films
}

\author{
A. E. Meyerovich and I. V. Ponomarev \\ Department of Physics, University of Rhode Island, Kingston, Rhode Island 02881 \\ (Received 29 October 2002; revised manuscript received 29 January 2003; published 16 April 2003)

\begin{abstract}
Conductivity of quantized multilayer metal films is analyzed with an emphasis on scattering by rough interlayer interfaces. Three different types of quantum size effect (QSE) in conductivity are predicted. Two of these QSE's are similar to those in films with scattering by rough walls. The third type of QSE is unique and is observed only for certain positions of the interface. The corresponding peaks in conductivity are very narrow and high with a finite cutoff which is due only to some other scattering mechanism or the smearing of the interface. There are two classes of these geometric resonances. Some of the resonance positions of the interface are universal and do not depend on the strength of the interface potential while the others are sensitive to this potential. This geometric QSE gradually disappears with an increase in the width of the interlayer potential barrier.
\end{abstract}

DOI: 10.1103/PhysRevB.67.165411

PACS number(s): 73.40.-c, 73.63.Hs, 72.10.Fk, 73.50.-h

\section{INTRODUCTION}

Boundary scattering is essential for a complete description of nanosystems such as quantum wells, ultrathin films or wires, etc. Due to the large surface-to-volume ratio, boundaries are expected to play a much greater role in determining the overall properties in a nanostructure than in a bulk material. For example, recent scanning tunneling microscopy (STM) data have shown that electron energy spectra can be more strongly correlated to the buried interfacial lattices than to the surface immediately beneath the STM tip. ${ }^{1}$ These observations clearly indicate that a small lateral variation along the boundary can have a significant long-range effect in a semiballistic electron system. Thus, a more realistic description of a nanoscale-quantized system must go beyond the common perfect geometric boundary and include boundary corrugations. Indeed, random surface roughness of a thin metal film can dominate incoherent scattering and relaxation, and can lead to an anomalous quantum size effect such as large oscillatory dependence of the in-plane conductivity on the film thickness. ${ }^{2}$

The same must be true not only for the quantum well (film) walls but also for the interlayer interfaces in multilayer films. It is well known that the roughness of the interlayer interfaces plays an important role in, for example, giant magnetoresistance (see the review in Ref. 3 and references therein). The purpose of this paper is to analyze the effect of irregular corrugation of the interlayer interfaces on the lateral conductivity of quantized multilayer films without magnetic effects. We will see that the interface scattering can result in unique features of the quantum size effect (QSE) which are strikingly different from the QSE with scattering by bulk or wall inhomogeneities. Orbital and spin magnetic effects of the type studied in Ref. 4 will be studied separately.

In ultrathin films, the motion of electrons across the films can be quantized. QSE in metal films is studied experimentally by measuring conductivity ${ }^{5,6}$ and susceptibility ${ }^{7}$ of the films or in spectroscopy ${ }^{8}$ and STM (Ref. 1) measurements (for earlier results, see references therein). As a result of the QSE, the three-dimensional (3D) electron spectrum $\epsilon(\mathbf{p})$ splits into a set of minibands $\epsilon_{j}(\mathbf{q})$ where $\mathbf{q}$ is the $2 \mathrm{D}$ mo- mentum along the film ( $y z$ plane). In the simplest case of a single-layer film approximated by a rectangular quantum well, the quantized values of the $x$ component of momentum are $p_{x_{j}}=\pi j / L$ (here and below $\hbar=1$ ). If in such quantized metal films the Fermi energy $E_{F}$ is unaffected by the quantization, the Fermi surface reduces to a set of 2D curves $\epsilon_{F j}(\mathbf{q})$ that correspond to cross sections of the 3D Fermi surface $\epsilon(\mathbf{p})=E_{F}$ by a set of planes $p_{x_{j}}=\pi j / L, \epsilon_{F j}(\mathbf{q})$ $=\epsilon_{F}\left(p_{x_{j}}, \mathbf{q}\right)$.

This quantization of motion, which is determined by the film thickness $L$, leads to several types of QSE. First, any change of the film thickness $L$ results in a change in the size and number of the Fermi curves $\epsilon_{F j}(\mathbf{q})$. This thicknessdriven change in number of the Fermi curves $\epsilon_{F j}(\mathbf{q})$ [or, what is the same, number of occupied minibands $\epsilon_{j}(\mathbf{q})$ ] leads to a singularity in the density of states. These singularities are the most obvious manifestations of QSE.

These singularities in the density of states, by themselves, do not lead to any striking anomalies in the dependence of the lateral conductivity $\sigma$ of the film on the thickness $L$. The conductivity is more sensitive to electron scattering than to the density of states. However, the change in the number of occupied minibands $S$ can be accompanied by a change in the number of allowed scattering channels that correspond to the scattering-driven electron transitions between minibands $\epsilon_{j}(\mathbf{q})$. The effect of this steplike change in the number of scattering channels on the conductivity is much stronger than that of the singularities in the density of states. ${ }^{9}$ When all scattering-driven interband transitions are allowed, the QSE manifests itself as a pronounced sawlike dependence of the conductivity on the film thickness. This type of QSE in quantized films has been predicted both for scattering by impurities and surface inhomogeneities. ${ }^{10,11}$

When the main scattering mechanism is the scattering by surface inhomogeneities, many of the interband transitions can often be suppressed. This happens, for example, when the average size of the surface inhomogeneities, $R$, is much larger than the the thickness of the film and/or the particle wavelength, $\lambda_{F}$. Then the usual QSE, which is described above, disappears and is replaced by a different kind of the 
size effect. ${ }^{2}$ This anomalous QSE, which is somewhat reminiscent of the magnetic breakthrough, is completely decoupled from the singularities in the density of states and is associated solely with opening of interband scattering channels for gliding electrons at certain values of the film thickness, $L_{i} \simeq \sqrt{(i+1 / 2) R \lambda_{F} / 2}$.

The main goal of this paper is to analyze QSE in multilayer films with an emphasis on the scattering by the interface between the layers. We will see that, in addition to the above two types of QSE, the multilayer films can exhibit a peculiar "geometric" QSE with very narrow high peaks in the lateral conductivity. Some of the positions of these spikes in conductivity are universal; these spikes appear when the ratio of the thicknesses of the film layers is given by simple fractions. The position of the rest of the spikes depends on the strength of the interlayer interface.

In the next section, we briefly present the main equations for the conductivity and introduce proper dimensionless variables. The results are presented in Sec. III. Section IV contains the summary and a brief discussion of the results. The Appendix contains auxiliary information on the energy spectrum of multilayer films of the type used in the calculations.

\section{CONDUCTIVITY}

\section{A. Scattering by the interlayer interface}

For simplicity, we consider an ultrathin film of thickness $L$ consisting of only two layers with the thickness of $L_{1}$ and $L_{2}$. The interface between the layers is rough with random corrugation. The exact position of the interface, $x=L_{1}$ $+\xi(y, z)$, is described by the random function $\xi(y, z)$ with zero average $\langle\xi\rangle=0$. The random interface inhomogeneities $\xi(y, z)$ are best characterized by the correlation function $\zeta(\mathbf{s})$

$$
\zeta(\mathbf{s}) \equiv \zeta(|\mathbf{s}|)=\left\langle\xi\left(\mathbf{s}_{1}\right) \xi\left(\mathbf{s}_{1}+\mathbf{s}\right)\right\rangle \equiv A^{-1} \int \xi\left(\mathbf{s}_{1}\right) \xi\left(\mathbf{s}_{1}+\mathbf{s}\right) d \mathbf{s}_{1},
$$

where the vector $\mathbf{s}$ gives the $2 \mathrm{D}$ coordinates along the interface and $A$ is the averaging area. Here, it is assumed that the correlation properties of the surface do not depend on direction. Two main characteristics of the surface correlation functions $\zeta$ are the average amplitude ("height") and the correlation radius ("size") of surface inhomogeneities, $\ell$ and $R$.

To emphasize the scattering by inhomogeneities of the interlayer interface, we start from films with ideal outside walls that do not contribute to electron scattering. The combined effect of interface and wall inhomogeneities will be considered elsewhere.

Mostly we are interested in the dependence of the lateral conductivity on the film thickness and have in mind the following experimental situation. The first layer of the film is grown on some (ideal) substrate. The surface is then roughened by adding inhomogeneous adsorbate or by some other means. The growth of the second layer starts from this rough interface, and the conductivity is measured at different values of $L_{2}$ either in the process of growth or after the growth is completed. An advantage of such setup with a buried interface is that it allows one to measure the conductivity at various values of the film thickness with exactly the same random rough interface.

In this setup, the thickness of the first layer, $L_{1}$, should be considered as fixed, while the thickness of the second layer, $L_{2}$, is variable. Below we calculate the film conductivity $\sigma$ as a function of the film thickness, $L=L_{1}+L_{2}, \sigma(L)$, assuming that $L_{1}=$ const. The measurements of conductivity can be performed in stationary conditions at different values of $L_{2}$ or as a function of time, in the process of film growth as in Ref. 12.

The second layer can be made of the same or different material as the first. If the material is different, then the electron potential energy between the layers differs by some $\Delta U$. The structure of the energy spectrum becomes a complicated function of $\Delta U$, making the behavior of conductivity highly irregular. ${ }^{13}$

Below we consider both layers to be made of the same material with the interface being the only disruption in the potential relief. Then the simplest model of the interface is the $\delta$-functional potential barrier

$$
U=U_{0} \delta\left(x-L_{1}-\xi(y, z)\right)
$$

This immediately introduces two new physical parameters into the problem: the strength of the barrier $U_{0}$ and its (average) position $L_{1}$. In what follows, we study the dependence of the conductivity on these parameters. When necessary, instead of the $\delta$ function we will study the corrugated interface with finite width $D$. In experiment, the barrier can be a dislocation wall, twin boundary, or an oxide or dielectric layer (see, e.g., Ref. 14 and references therein).

The presence of the interface (2) changes the spectrum. When calculating the changes in the spectrum, one can ignore small corrugation $\xi(y, z)$. The changes in spectrum caused by the $\delta$-type barrier (2) are discussed in the Appendix. The random corrugation of the interface is responsible for the electron scattering and gives rise to the collision operator in the transport equation.

The scattering by the interface inhomogeneities leads to the transitions between the states $\epsilon_{i}(\mathbf{q}) \rightarrow \epsilon_{j}\left(\mathbf{q}^{\prime}\right)$. Several ways of calculating the corrugation-driven transition probabilities $W_{i j}\left(\mathbf{q}, \mathbf{q}^{\prime}\right)$ are described in Ref. 13. The simplest methods are either the direct perturbation approach ${ }^{15}$ or the mapping transformation method, ${ }^{16}$ both giving the same result in most of the parameter range.

The corrugation-driven contribution $\delta U$ to the interface potential, Eq. (2), with small corrugation $\xi$ is

$$
\delta U=-U_{0} \xi(y, z) \delta^{\prime}\left(x-L_{1}\right) .
$$

The matrix element $V_{i j}\left(\mathbf{q}, \mathbf{q}^{\prime}\right)$ of this perturbation between the states $\epsilon_{j}(\mathbf{q}), \epsilon_{j}\left(\mathbf{q}^{\prime}\right)$ is 


$$
\begin{aligned}
V_{i j}= & -U_{0} \int \exp \left[i \mathbf{s} \cdot\left(\mathbf{q}-\mathbf{q}^{\prime}\right)\right] \xi(\mathbf{s}) \Psi_{i}(x) \delta^{\prime}\left(x-L_{1}\right) \\
& \times \Psi_{j}(x) d x d \mathbf{s} \\
= & U_{0} \xi\left(\mathbf{q}-\mathbf{q}^{\prime}\right)\left[\Psi_{i}\left(L_{1}\right) \Psi_{j}^{\prime}\left(L_{1}\right)+\Psi_{i}^{\prime}\left(L_{1}\right) \Psi_{j}\left(L_{1}\right)\right],
\end{aligned}
$$

where $\Psi_{i}(x)$ are the quantized wave functions for electron motion across the film. Note that the derivatives $\Psi^{\prime}(x)$ for films with a $\delta$-type barrier inside are discontinuous at the position of the barrier, $x=L_{1}$. Therefore, $\Psi_{i}^{\prime}\left(L_{1}\right)$ in Eq. (4) should be understood as $\Psi_{i}^{\prime}\left(L_{1}\right)=\left[\Psi_{i}^{\prime}\left(L_{1}+0\right)+\Psi_{i}^{\prime}\left(L_{1}\right.\right.$ $-0)] / 2$.

The corrugation-driven transition probability $W_{i j}\left(\mathbf{q}, \mathbf{q}^{\prime}\right)$ is given by the square of this matrix element which should be averaged over the random inhomogeneities $\xi$ :

$$
\begin{gathered}
W_{i j}\left(\mathbf{q}, \mathbf{q}^{\prime}\right)=\left\langle\left|V_{i j}\left(\mathbf{q}, \mathbf{q}^{\prime}\right)\right|^{2}\right\rangle_{\xi}=U_{0}^{2} \zeta\left(\left|\mathbf{q}_{i}-\mathbf{q}_{j}^{\prime}\right|\right) G_{i j}, \\
G_{i j}=\left[\Psi_{i}\left(L_{1}\right) \Psi_{j}^{\prime}\left(L_{1}\right)+\Psi_{i}^{\prime}\left(L_{1}\right) \Psi_{j}\left(L_{1}\right)\right]^{2},
\end{gathered}
$$

where $\zeta\left(\left|\mathbf{q}_{i}-\mathbf{q}_{j}^{\prime}\right|\right)$ is the Fourier image of the correlation function of the interface inhomogeneities (1). The coefficients $G_{i k}$ are calculated with the help of the wave functions presented in the Appendix. The explicit form of $G_{i k}$ is given in the next subsection.

The transport equation is a set of equations for the electron distribution functions $n_{i}(\mathbf{q})$ in minibands $\epsilon_{i}$ and has the standard Boltzmann-Waldmann-Snider form ${ }^{13}$

$$
\frac{d n_{i}}{d t}=2 \pi \sum_{j} \int W_{i j}\left[n_{j}-n_{i}\right] \delta\left(\boldsymbol{\epsilon}_{i \mathbf{q}}-\boldsymbol{\epsilon}_{j \mathbf{q}^{\prime}}\right) \frac{d^{2} q^{\prime}}{(2 \pi)^{2}}
$$

The integration over $d q^{\prime}$ is done using the $\delta$ function $\delta\left(\boldsymbol{\epsilon}_{i \mathbf{q}}-\boldsymbol{\epsilon}_{j \mathbf{q}^{\prime}}\right)=m_{i j}^{*} \delta\left(q^{\prime}-q_{i j}\right) / q_{i j}$, where $q_{i j}(q)$ is the solution of the equation $\epsilon_{j}\left(\mathbf{q}_{i j}\right)=\epsilon_{i}(\mathbf{q})$ and the effective masses $m_{i j}^{*}=q_{i j} /\left.\left(\partial \epsilon_{j} / \partial q\right)\right|_{q=q_{i j}}$. As always in the transport theory, the angular integration is eliminated by using the angular harmonics. The current is given by the first harmonic of the distribution $n_{i}^{(1)} \equiv \nu_{i}$ the equation for which involves only the zeroth and first harmonics $W_{i j}^{(0,1)}\left(q, q_{i j}\right)$ of $W\left(\mathbf{q}-\mathbf{q}_{i j}\right)$ over the angle $\widehat{\mathbf{q q}}_{j j^{\prime}}$,

$$
\begin{gathered}
d \nu_{i}(q) / d t=-\sum_{j} \nu_{j}\left(q_{i j}\right) / \tau_{i j}, \\
\frac{1}{\tau_{i j}}=\frac{m}{2} \sum_{k}\left[\delta_{i j} W_{i k}^{(0)}-\delta_{j k} W_{i j}^{(1)}\right], \\
W_{i j}^{(0,1)}=U_{0}^{2} \zeta^{(0,1)}\left(\mathbf{q}_{i}-\mathbf{q}_{j}\right) G_{i j},
\end{gathered}
$$

where, to simplify the equations, we assume that the effective mass $m_{i j}^{*}$ does not depend on its indices, $m=m_{i j}^{*}$.

The solution of Eqs. (8) provides the 2D conductivity of the film:

$$
\sigma=-\frac{e^{2}}{3 \hbar^{2}} \sum_{i} \nu_{i}\left(q_{i}\right) q_{i}
$$

\section{B. Dimensionless variables}

The problem involves several length scales: particle Fermi wavelength $\lambda_{F}=\pi / p_{F}$; the thickness of the layers, $L_{1}$ and $L_{2}\left(L_{1}+L_{2}=L\right)$; the correlation radius of the surface inhomogeneities, $R$; and the interface thickness $D$. Another length parameter, the amplitude of inhomogeneities $\ell$, is perturbative and enters conductivity as a coefficient,

$$
\sigma=\frac{2 e^{2}}{\hbar} \frac{R^{2}}{\ell^{2}} f\left(\lambda_{F}, L_{i}, R, D\right) .
$$

Note that we consider only the contribution from surface roughness and disregard the bulk scattering. As a result, the conductivity (10) diverges in the limit of vanishing inhomogeneities $\ell \rightarrow 0$ or $R \rightarrow \infty$. The proper account of bulk scattering ${ }^{17}$ eliminates this divergence.

It is convenient to measure all length parameters in units of the Fermi wavelength $\lambda_{F}=\pi / p_{F}$. Instead of the interface strength $U_{0}$, we use interchangeably two equivalent dimensionless parameters $g$ and $u_{0}$,

$$
g=u_{0} L / \pi \lambda_{F}=2 m U_{0} \lambda_{F} L / \pi \hbar^{2}
$$

( $g$ is convenient for calculation of the spectrum while $u_{0}$ is a proper energy parameter for characterization of the conductivity in our setup). The position of the interface is characterized by the parameter $\delta$,

$$
\delta=L_{2} / L \text {. }
$$

In computations, $\delta$ changes from 0 (no second layer) to 1 (the second layer much wider than the first). It is worth repeating that we are looking at the experimental situation when the thickness of the first layer is fixed and the conductivity is measured as a function of the thickness of the second layer (or the overall film thickness).

The energy spectrum $\epsilon_{i}(\mathbf{q})$ is described by dimensionless energy units $z_{i}$,

$$
\boldsymbol{\epsilon}_{i}(\mathbf{q})=\frac{1}{2 m}\left(\frac{\pi^{2}}{L^{2}} z_{i}^{2}+q^{2}\right),
$$

where $z_{i}$ is given by the solution of the 1D Schrödinger equation for a quantum well with a $\delta$-type barrier inside (see the Appendix):

$$
\sin \pi z+\frac{g}{z} \sin (\pi z \delta) \sin [\pi z(1-\delta)]=0 .
$$

Finally, the conductivity $\sigma(L)$ for the experimental setup, which has been described above, will be displayed by the dimensionless function $f_{L}\left(L / \lambda_{F}\right)$,

$$
\sigma(L)=\frac{2 e^{2}}{\hbar} \frac{R^{2}}{\ell^{2}} f_{L},
$$

for various values of $R / \lambda_{F}, D / \lambda_{F}, L_{1} / \lambda_{F}$, and the strength of the barrier $u_{0}$.

All the figures below present this dimensionless function $f_{L}$. This function is plotted under the assumption that the 
experiment is performed at fixed thickness of the first layer. For uniformity, the figures for weak interfaces are plotted for $u_{0}=0.1$ and, for strong interface barriers, for $u_{0}=10$. The simplest energy spectrum corresponds to thin first layers, $\lambda_{F} \leqslant L_{1}<2 \lambda_{F}$. Therefore, for transparency of results, the majority of the data are presented for $L_{1} / \lambda_{F}=1.1$ (for comparison, some of the graphs give the conductivity for larger $\left.L_{1}\right)$.

The computational results below are presented for the Gaussian correlation function of the interface inhomogeneities,

$$
\zeta(\mathbf{s})=\ell^{2} \exp \left(-s^{2} / 2 R^{2}\right) .
$$

The angular harmonics for this correlator, which enter the transition probabilities in Eq. (8), are equal to

$$
\begin{aligned}
& \zeta^{(0)}\left(q_{i}, q_{j}\right)=4 \pi \ell^{2} R^{2}\left[e^{-Q Q^{\prime}} I_{0}\left(Q Q^{\prime}\right)\right] e^{-\left(Q-Q^{\prime}\right)^{2} / 2}, \\
& \zeta^{(1)}\left(q_{i}, q_{j}\right)=4 \pi \ell^{2} R^{2}\left[e^{-Q Q^{\prime}} I_{1}\left(Q Q^{\prime}\right)\right] e^{-\left(Q-Q^{\prime}\right)^{2} / 2},
\end{aligned}
$$

where $Q=q_{i} R, Q^{\prime}=q_{j} R$.

Analysis of QSE in Ref. 2 for ultrathin films with scattering by the film walls demonstrated that the results for all types of correlators are qualitatively the same as for the Gaussian one when $R \ll L$. For large inhomogeneities $R$ $\gg L$, the results for all types of correlators with exponential power spectra are similar to those for the Gaussian correlator and are qualitatively different from the power-law correlators. The results for the power-law correlators are less interesting: such films always exhibit the standard sawlike QSE irrespective of the value of $R$ because of the wider fluctuations of the inhomogeneity sizes. Therefore, in this paper we consider only the exponential correlators with a well-defined size of inhomogeneities.

\section{RESULTS}

\section{A. Standard quantum size effect}

The standard quantum size effect in films manifests itself by a sawlike dependence of the conductivity $\sigma$ on the film thickness $L^{10,11}$ The positions of the singularities-the sawteeth-correspond to the values of the thickness at which a new energy miniband $\epsilon_{j}$ becomes accessible. The amplitude of the conductivity drop in such a singular point depends, in the case of scattering by surface inhomogeneities, on the effectiveness of the roughness-driven interband transitions. If the probability of such transitions $W_{i \neq j}$, is small in comparison to the rate of the intraband scattering $W_{i i}$, the singularities in the curves $\sigma(L)$ are almost completely suppressed and the standard QSE disappears. ${ }^{9}$

Analysis of the roughness-driven transition probabilities for surface scattering in Ref. 2 for different classes of surface roughness showed that, when the average size of inhomogeneities, $R$, is much smaller than the film thickness $L$, the values of the interband transition probabilities $W_{i \neq j}$ are comparable to that for the intraband scattering $W_{i i}$ and all scattering channels are equally important. In this case, the curves $\sigma(L)$ always exhibit the standard QSE. The same should be

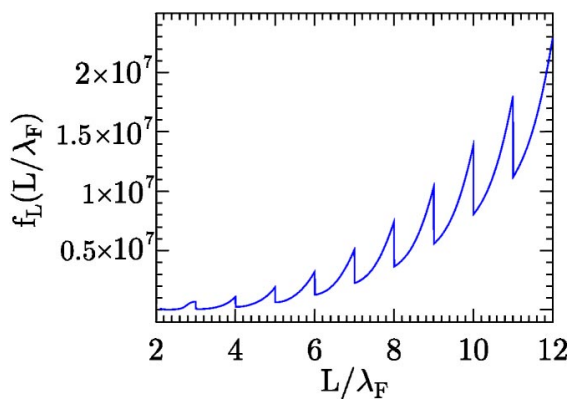

FIG. 1. Dimensionless conductivity of quantized films, Eq. (15), as a function of the film thickness $L$. The sawlike dependence is typical for the standard quantum size effect. The correlation radius of inhomogeneities, $R / \lambda_{F}=1$; the thickness of the first layer, $L_{1} / \lambda_{F}=2.1$; the width of the interface, $d=D / \lambda_{F}=0.01$; and the strength of the barrier, $u_{0}=0.1$.

true for scattering by the interlayer interfaces. This is illustrated in Figs. 1 and 2 which show $\sigma(L)$ for a weak and strong interface potentials $u_{0}=0.1$ and $u_{0}=10$ respectively. In Fig. 1 the thickness of the first layer is $L_{1}=2.1$ and $\lambda_{F}$, in Fig. 2, $L_{1}=1.1 \lambda_{F}$. In both figures, the size of inhomogeneities is $R=\lambda_{F}$. Both figures exhibit a well-pronounced sawlike structure. The positions of the singularities for the weak interface are almost equidistant, reflecting the fact the energy structure is close to that for a square well without perturbation inside. The strong interface affects the energy spectrum and, therefore, the positions and the shapes of the sawteeth. However, at very large film thickness $L \gg L_{1}$ the interface is located very close to the well wall and the spectrum starts to recover its unperturbed structure. This manifests itself in a recovery of the equidistant distribution of the singularities in Fig. 2 at large $L$. Because of a peculiar dependence of the transition probabilities on the interface strength (see the Appendix), the conductivity grows much faster with increasing film thickness in the case of the weak interface than for the strong interface.

\section{B. Quantum size effect for large-scale inhomogeneities}

The standard QSE of the type described in the previous subsection disappears in the single-layer film when the correlation size of inhomogeneities, $R$, is larger than the film

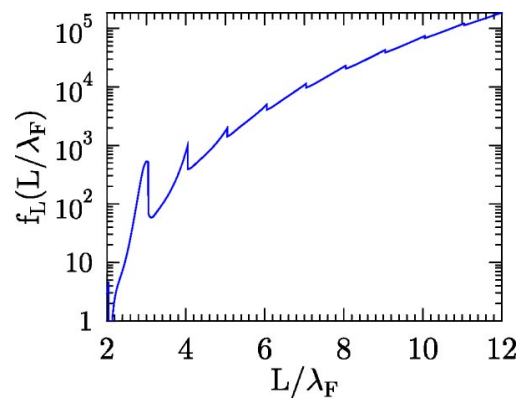

FIG. 2. Standard QSE in conductivity of quantized films, Eq. (15), as a function of the film thickness $L$ for strong interface potential, $u_{0}=10$. The correlation radius of inhomogeneities, $R / \lambda_{F}$ $=1$; the thickness of the first layer, $L_{1} / \lambda_{F}=1.1$; and the width of the interface, $d=10^{-4}$. 
thickness, $R \gg L$, and the correlation function in the momentum space, $\zeta(\mathbf{q})$ (the so-called power spectrum of inhomogeneities), decays exponentially at large wave numbers q. Instead, the single-layer films exhibit an anomalous QSE. ${ }^{2}$

The explanation involves the interband transitions. It seems that at large $R$ the off-diagonal $W_{i \neq k}$ are small and the interband transitions are suppressed. However, at certain values of large $L$, few of the elements $W_{i \neq k}$, which are close to the main diagonal, could become comparable to $W_{i i}$ even for large $R$. Then the transitions $i \leftrightarrow i+1$ could become noticeable, leading to a drop in conductivity. A simple estimate of the peak positions is the following. Scattering by surface inhomogeneities changes the tangential momentum by $\Delta q$ $\sim \pi / R$. This is sufficient for the interband transition when $\Delta q \sim q_{i}-q_{i+1}$. When the number of occupied minibands is large, the lateral Fermi momentum for the gliding electrons, i.e., electrons from the miniband with a relatively small in$\operatorname{dex} i, q_{i} \sim p_{F}$. For such electrons, $q_{i}^{2}-q_{i+1}^{2} \sim 2 \pi \Delta q / \lambda_{F}$ $\sim 2 \pi^{2} / R \lambda_{F}$. On the other hand, the energy conservation law dictates $q_{i}^{2}-q_{i+1}^{2}=(2 i+1) \pi^{2} / L^{2}$. Accordingly, with increasing $L$ the transition channel $i \leftrightarrow i+1$ opens at $L^{2} \sim(i$ $+1 / 2) R \lambda_{F}$. The opening of a new scattering channel in the points

$$
L_{i} \sim \sqrt{(i+1 / 2) R \lambda_{F}}
$$

is always accompanied by a drop in conductivity. The first such drop occurs for the electrons in the lowest miniband $\epsilon_{1}(\mathbf{q})$ with $i=1$, i.e., for the grazing electrons. Note that these particular electrons contribute the most to the conductivity. Since the electrons from the lowest miniband are responsible for the dominant contribution to the conductivity, the conductivity drops almost by half in the point $L_{1}$ $\sim \sqrt{3 R \lambda_{F} / 2}$ where $W_{12}$ becomes comparable to $W_{11}$ and the effective cross section doubles. (In the quasiclassical film without bulk scattering, the current, which is an integral over momenta, diverges when the component of momentum perpendicular to the film goes to zero, i.e., for the grazing electrons. Without the bulk scattering, the conductivity is finite only because of the quantum cutoff at $p_{x}=\pi / L$.)

The anticipation was that this type of QSE should manifest itself also for the interface scattering in multilayer films at $R \gg L$ for exponentially decaying surface correlators. Indeed, such a picture can be observed in Figs. 3 and 4 for $u_{0}=0.1,10$, respectively (in both figures, $L_{1}=1.1 \lambda_{F}, R$ $=200 \lambda_{F}$ ). The positions of the peaks in Fig. 3 for the weak interface are close to Eq. (18). In the case of the strong interface, the shift of the energy levels from those for an "empty" square well is much more noticeable and the positions of the peaks in Fig. 4 deviate from those given by Eq. (18). At large values of $L$, the positions of the peak with strong interface become close to the points in which the thickness of the second layer, $L_{2}=L-L_{1}$, rather than the overall thickness $L$ is given by Eq. (18). The amplitude of the anomalous QSE oscillations grows with the increasing strength of the interface approaching that for the impenetrable wall.

Of course, for the inhomogeneities of the intermediate size, the picture exhibits the features of both standard and

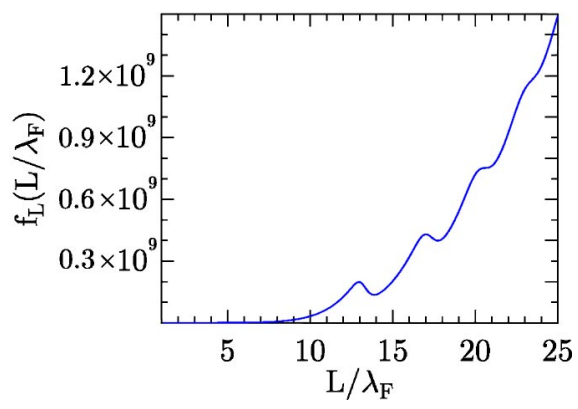

FIG. 3. Anomalous QSE in conductivity of quantized films, Eq. (15), as a function of the film thickness $L$. The correlation radius of inhomogeneities is large, $R / \lambda_{F}=200$; the thickness of the first layer, $L_{1} / \lambda_{F}=2.1$; the width of the interface, $d=0.1$; and the strength of the interface barrier, $u_{0}=0.1$.

anomalous QSE's. As has already been mentioned, our numerical examples address the experiment in which the size of the inhomogeneities, $R$, is fixed while the thickness of the film, $L$, is changing. In general, at the values $L<R$ one should see the smooth anomalous QSE oscillations with large period, while at $L>R$ one should, on the same curve, see the reappearance of the standard QSE with sharper oscillations with period equal to 1 . Roughly, the transitions between the regimes occurs when the distance between the peaks of the anomalous QSE, Eq. (18), decreases to the value $\left(L_{i+1}-L_{i}\right) / \lambda_{F} \sim 1$. In principle, the reappearance of the standard QSE should be seen in Figs. 3 and 4 when the computations are extended to sufficiently large $L$. However, the amplitude of the standard QSE oscillations on these curves is very small and the reappearance of the oscillations is barely noticeable on the scale of the curve. It is much more illustrative to demonstrate the effect at intermediate values of $R$ when both anomalous and standard QSE oscillations have comparable amplitude. This is shown in Fig. 5 for $R / \lambda_{F}$ $=3$ and weak interface $u_{0}=0.1$. On the left side of the graph one can clearly see smooth "new" oscillations with a relatively large period, while on the right side the oscillations recover the sharp sawlike structure with period equal to 1 .

\section{Geometric (fractional) quantum size effect}

To exhibit the QSE oscillations of the previous subsection, Figs. 3 and 4 were plotted not for the exact $\delta$-type interfaces (2) and (3) but for a somewhat smeared (less sharp) interface

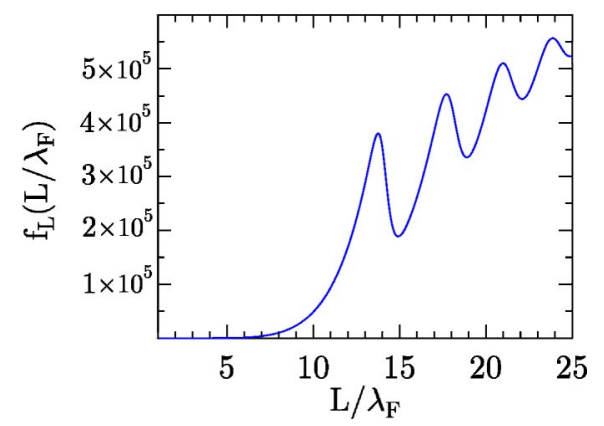

FIG. 4. Same as in Fig. 3, but for a much stronger interface barrier, $u_{0}=10$. 


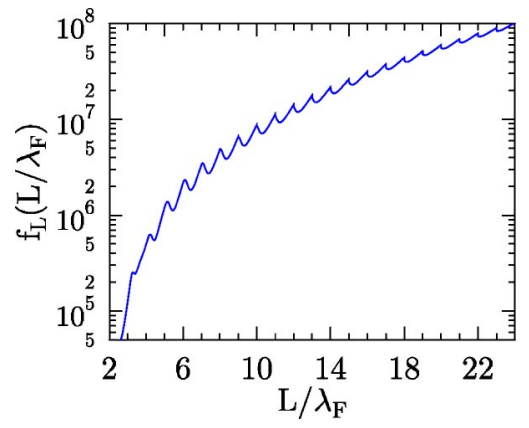

FIG. 5. QSE in conductivity of quantized films, Eq. (15), as a function of the film thickness $L$ for the intermediate values of the size of inhomogeneities, $R / \lambda_{F}=3$. The thickness of the first layer, $L_{1} / \lambda_{F}=1.1$; the width of the interface, $d=0.1$; and the interface barrier, $u_{0}=0.1$. At small $L$, the curve exhibits the smooth oscillations of the anomalous QSE with a large period, while QSE for large $L$ recovers the standard sawlike shape with period equal to 1 .

$$
\delta U=-U_{0} \xi(y, z)\left[\delta^{\prime}\left(x-L_{1}\right)+D \delta^{\prime \prime}\left(x-L_{1}\right)\right] .
$$

The interface width $D$ can have two origins. If its origin is corrugation related, then the interface width is given by the next term of expansion of the interface barrier in $\xi$ and is characterized by the same parameters $\ell$ and $R, D^{2} \sim\left\langle\xi^{2}\right\rangle$. In this case, depending on the correlation function, $D \sim \ell$ or $D \sim \ell^{2} / R$. On the other hand, $D$ can originate from some "internal" smearing of the interface and can exist even without surface inhomogeneities. In this case, $D$ is a new independent small parameter. Note that here we are interested in the "smearing" of the interface and not in its "fixed" width so that the average of the square of the matrix elements of $\delta U$ over the interface starts from $D^{2}$. In Figs. 3 and 4 , the interface thickness was chosen as $d=D / \lambda_{F}=0.1$.

If the interface is thinner, the character of the curves changes dramatically. For example, Fig. 6 presents the conductivity $\sigma(L)$ exactly for the same values of all parameters as in Fig. 3 except for the interface thickness which is now $d=D / \lambda_{F}=0.0001$. The difference between the two curves is astonishing.

The conductivity in Fig. 6 exhibits two types of spikes. The explanation for first type of spikes is the following. The scattering-driven transition probabilities $W_{i j}$, Eq. (5), contain the factor with the correlation function $\zeta\left(\mathbf{q}_{i}-\mathbf{q}_{j}^{\prime}\right)$ and

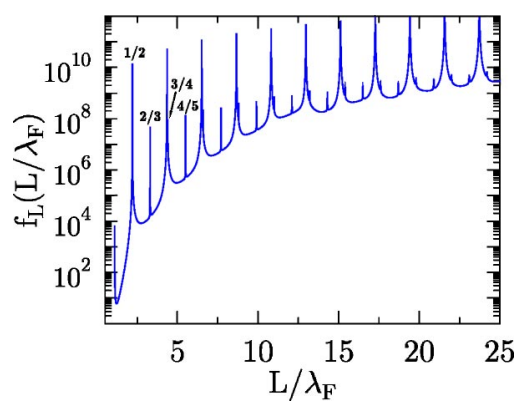

FIG. 6. Geometric QSE in conductivity of multilayer films. The same parameters as in Fig. 3, except for a much sharper interface, $d=10^{-4}$. The fractions near the spikes give the values of the resonance positions of the interface $\delta=L_{2} / L$. the geometric coefficients $G_{i j}$, Eq. (6). For exponentially decaying correlators with large $R \gg L$, the off-diagonal values of the correlation function $\zeta\left(\mathbf{q}_{i}-\mathbf{q}_{j}^{\prime}\right)$ with $i \neq j$ are exponentially small in comparison with the diagonal ones, $\zeta\left(\mathbf{q}_{i}\right.$ $-\mathbf{q}_{i}^{\prime}$ ). Then it is sufficient to analyze only the diagonal elements of the matrix $G_{i j}$, Eq. (6):

$$
G_{i i}=4 \Psi_{i}^{2}\left(L_{1}\right) \Psi_{i}^{\prime 2}\left(L_{1}\right)
$$

If, accidentally, the $\delta$-type interface is positioned in the points in which either $\Psi_{i}\left(L_{1}\right)=0$ or $\Psi_{i}^{\prime}\left(L_{1}\right)=0$, then the coefficient $G_{i i}$ and, therefore, the transition probability $W_{i i}$ become zero. This, in turn makes the conductivity of electrons in the miniband $\epsilon_{i}$ and, therefore, the overall conductivity almost infinite. The cutoff is determined by one of three factors: (1) exponentially small interband transitions, (2) scattering by other defects such as impurities, inhomogeneities of external walls, etc., and (3) smearing of the interface, (19), which leads to the averaging of $G_{i i}$, Eq. (6) and (20), over a finite interval, making it nonzero. In this paper, for obvious reasons, we are interested in the third option. Note that in the case of scattering by external film walls instead of the interlayer interface, the coefficients $G_{i j} \sim i^{2} j^{2}$ are never equal to zero and this type of QSE does not exist.

The first type of spikes corresponds to $\Psi_{i}\left(L_{1}\right)=0$. The "resonance" positions of the $\delta$-type interface are universal and do not depend on the potential strength. This is true for all rational points $\delta=L_{2} / L$. Of course, the conductivity of the film becomes infinite for this position of the interface only if the corresponding miniband $\epsilon_{i}$ is occupied. This means that the integer $n$ in the denominator of the corresponding fraction $\delta=m / n$ should not exceed the number of the occupied minibands, $n \leqslant S=\operatorname{Int}\left[L / \lambda_{F}\right]$. Indeed, for points $\delta=L_{2} / L=m / n$ there is a number of wave functions $\Psi_{i}(x)$ of the empty well that have nodes in the points $x$ $=L_{1}$. Since the unperturbed homogeneous potential barrier has a $\delta$-functional form $U_{0} \delta\left(x-L_{1}\right)$, these wave functions $\Psi_{i}(x)$ remain the eigenfunctions of the well with the unperturbed barrier $U_{0} \delta\left(x-L_{1}\right)$ inside and retain their nodes in the points $x=L_{1}$. Then the corresponding diagonal coefficients $G_{i i}$ are zero, making the diagonal roughness-driven transition probabilities $W_{i i}$ for particles from the miniband $\epsilon_{i}$ equal to zero as well. Since the off-diagonal transition probabilities are exponentially small in $R / L \gg 1$, the condition $W_{i i}=0$ makes the conductivity for particles from the miniband $\epsilon_{i}$, and, therefore, the overall conductivity exponentially large in $R / L \gg 1$.

The structure of the corresponding resonance spikes becomes more and more complicated with an increase in $L_{1}$ when the structure of the minibands and their occupancy become more convoluted. The simplest structure is observed when $L_{1}$ is between $\lambda_{F}$ and $2 \lambda_{F}$ as in Fig. 6 . In this case, the observed rational spikes correspond to the rational numbers of the form $\delta=(n-1) / n$ and are equidistant with the separation $L_{1} / \lambda_{F}$. The first spike corresponds to the film with $\delta=L_{2} / L=1 / 2$, the second to $\delta=2 / 3$, the third to $\delta=3 / 4$, the fourth to $\delta=4 / 5$, and so on. The odd peaks, with the exception of the first one, look wider and consist of bigger and smaller subpeaks. The smaller subpeaks correspond to the 


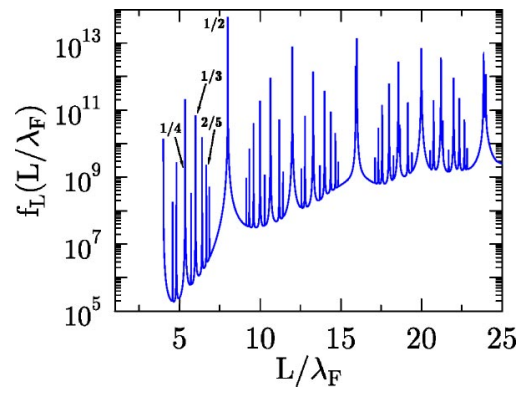

FIG. 7. Geometric QSE in conductivity of multilayer films. The same parameters as in Fig. 6, except for a wider first layer, $L_{1} / \lambda_{F}$ $=4.0$. The fractions near the spikes give the values of the resonance positions of the interface $\delta=L_{2} / L$.

geometrical resonance with $\delta=(n-1) / n$ which is described above. The bigger and wider subpeaks have a somewhat different nature and are not universal with respect to the barrier strength. These subpeaks will be described later. Note that the peak $\delta=3 / 4$ is so close to the first peak from the other series that these two peaks are hardly distinguishable.

When $L_{1}$ becomes bigger, the first few geometric resonances can be observed at much narrower second layers, well before the point $\delta=L_{2} / L=1 / 2$, while the density of the resonances becomes higher. For example, Fig. 7 presents the conductivity as a function of thickness for the film with the same parameters as in Fig. 6 except for the thickness of the first layer which is now $L_{1}=4 \lambda_{F}$. Though the overall distribution of the peaks is now much more complicated, the majority can still be understood as the ones generated by the eigenfunctions of the empty quantum well with the nodes in the positions of the barrier. The complexity of the peak structure is explained by the fact that at a wider first layer $L_{1}$ more minibands are occupied, thus allowing a wider selection of the rational numbers that determine the peak positions $\delta=L_{2} / L=m / n$.

The geometric resonances can coexist with the anomalous QSE of the previous section if the interface is relatively strong as in Fig. 8 for the same configuration as in Fig. 6 but with much higher value of $u_{0}, u_{0}=10$. For weak interfaces, the geometric resonances suppress the QSE of the previous subsection which gets restored only for bigger values of the

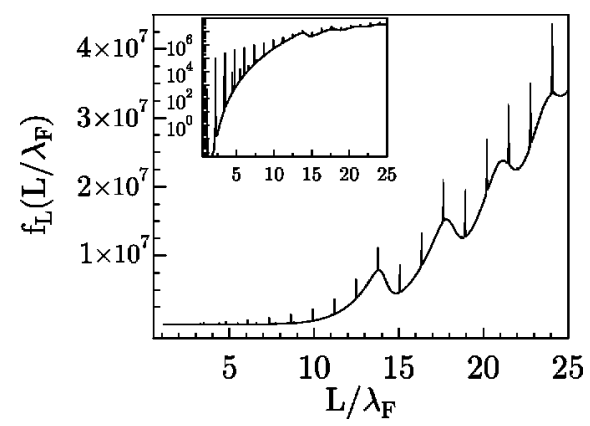

FIG. 8. Geometric and anomalous QSE in conductivity of multilayer films for strong interface potential, $u_{0}=10$. The rest of the parameters are the same parameters as in Fig. 6. For easier comparison with Figs. 4 and 6, the inset gives the same data in logarithmic scale.

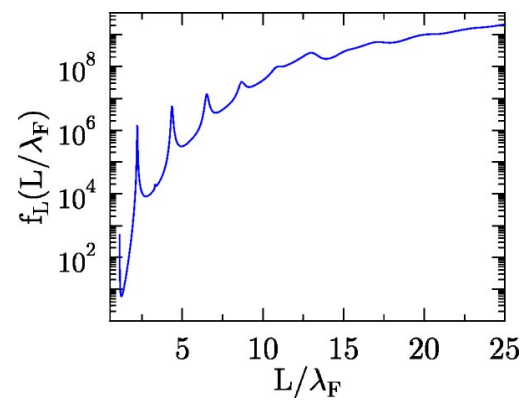

FIG. 9. Illustration of the effect of smearing of the interface. The same curve as in Fig. 6, but for a wider interface, $d=0.01$.

interface thickness $d$. This graduate disappearance of the geometric resonances can be seen when comparing Fig. 6 for $u_{0}=0.1, d=10^{-4}$ with Fig. $9\left(d=10^{-2}\right)$ and Fig. $10(d$ $\left.=10^{-1}\right)$. Figure 10 presents conductivity for the same configuration as Fig. 3 but in logarithmic scale. In this scale, one can see both the wide QSE oscillations of the previous subsection and the only surviving geometric resonance at $\delta=1 / 2$.

Above we explained only the narrower, universal geometric resonances at $\delta=m / n$ in Figs. 6-9. The second, nonuniversal type of spikes has a similar explanation: zeros of $G_{i i}$. According to the Appendix,

$$
\begin{aligned}
G_{i i} & =4 \Psi_{i}^{2}\left(L_{1}\right) \Psi_{i}^{\prime 2}\left(L_{1}\right) \\
& =\frac{4 \pi^{2}}{L^{4}} A_{i}^{4} \sin ^{2}\left(\pi z_{i} \delta\right)\left[g \sin \left(\pi z_{i} \delta\right)+2 z_{i} \cos \left(\pi z_{i} \delta\right)\right]^{2},
\end{aligned}
$$

where $z_{i}(g, \delta)$ is given by the solution of the 1D Schrödinger equation (14) for a quantum well with a $\delta$-type barrier inside. The explicit form of the coefficients $A_{i}$ is not important. The factor $\sin ^{2}\left(\pi z_{i} \delta\right)$ in Eq. (21) corresponds to $\Psi_{i}^{2}\left(L_{1}\right)$; its zeros are responsible for the geometric resonances with rational $\delta=(n-1) / n$. There are no other zeros of $\sin ^{2}\left(\pi z_{i} \delta\right)$.

However, Eq. (21) also contains the factor in the square brackets which corresponds to $\Psi_{i}^{\prime}\left(L_{1}\right)$. The simultaneous solution of Eq. (14) for the spectrum, $g \sin (\pi z \delta)$ $+2 z \cos (\pi z \delta)=0$, and equation $\Psi_{i}^{\prime}\left(L_{1}\right)=0$ yields the following equation for the resonance positions of the interface:

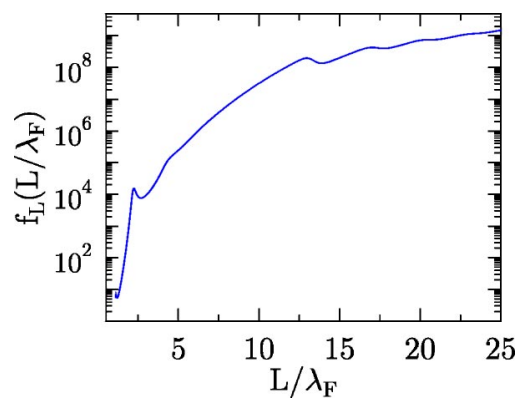

FIG. 10. Illustration of the effect of smearing of the interface. The same curve as in Figs. 6 and 9, but for an even wider interface, $d=0.1$. Data as in Fig. 3, but in logarithmic scale. 


$$
\sin \left[\pi z_{i}(1-2 \delta)\right]=0,
$$

which is equivalent to

$$
z_{i}(g, \delta)(1-2 \delta)=k,
$$

with integer $k$. First, there is a universal solution $\delta=1 / 2$ at $k=0$. In this case, when the interface is exactly in the middle of the film, both $\Psi_{i}\left(L_{1}\right)$ and $\Psi_{i}^{\prime}\left(L_{1}\right)$ are zero (the former with an even index, the latter with an odd index). This explains why the geometric resonance with $\delta=1 / 2$ is the most stable one with respect to the smearing of the interface.

The rest of the resonances with $k \neq 0$ are not universal. These resonances explain the earlier unaccounted for spikes in Figs. 6-8. Since the spectrum $z_{i}(g, \delta)$ is a complicated function of the interface strength and its position, the solution of Eq. (23) for $k \neq 0$ is rather complicated. We will give the analytic equation for the simplest case of $\lambda_{F} \leqslant L_{1}<2 \lambda_{F}$ when Eq. (23) is equivalent to

$$
\frac{L}{\lambda_{F}}=\frac{2 z_{n}}{z_{n}-n+1} \frac{L_{1}}{\lambda_{F}}
$$

(the only allowed values of $k$ are $k=-n+1$ ). For weak interfaces $g / z_{n} \ll 1$ this equation can be rewritten as

$$
\frac{1}{1-\delta}=2 n\left[1-\frac{n-1}{n} \Delta_{n}\right], \quad \Delta_{n} \approx \frac{g}{\pi z_{n}} \sin ^{2}(\pi n \delta) .
$$

Analysis of Eq. (25) shows that several of the first of such resonance positions of the interface are indeed close to the odd rational universal resonances as in Fig. 6,

$$
\delta \simeq \frac{2 l-1}{2 l}
$$

and separate from the universal resonances with increasing integer $l$. The very first resonance at $\delta=1 / 2$ is, as is explained above, exactly the same as the first universal resonance. The reason why these nonuniversal resonances are wider and stronger than the universal ones described above is still unclear.

\section{CONCLUSIONS}

In summary, we analyzed the QSE in the conductivity of multilayer films when the main scattering mechanism is the scattering of electrons by random inhomogeneities of the interlayer interface. Three different types of QSE are predicted.

The first one is a standard QSE with a typical sawlike dependence of the conductivity $\sigma$ on the film thickness $L$, $\sigma(L)$. This effect dominates when the correlation radius (size) of the interface inhomogeneities $R$ is much smaller than the film thickness, $R \ll L$. This effect should be observed for all types of correlation functions of the interface roughness. This effect is easily explained by the singularities in the electron density of states related to the quantization of motion across the film.

The second type of QSE is explained not by the quantization-driven singularities in the density of states, but by the anomalies in the cross section for scattering by interface inhomogeneities. This scattering-driven QSE replaces the standard sawlike QSE when the correlation radius (size) of the interface inhomogeneities is large, $R \gg L$. This type of QSE manifests itself as smooth large-scale oscillations on the dependence $\sigma(L)$ and should be observed only when the Fourier image of the interface correlation function (the socalled power spectrum of inhomogeneities) decays exponentially at large momenta. The main difference of this QSE from a similar effect in scattering by the film walls ${ }^{2}$ is that the observation of this effect in multilayer film requires certain smearing of the interface.

The third type of QSE is new and is most unusual. This effect manifests itself as a set of very narrow and high spikes in $\sigma(L)$ and replaces the scattering-induced QSE described above when the interface is narrow. The finite cutoff in the spikes can be ensured either by some other scattering mechanism or by the smearing of the interface.

The spikes are observed only for certain resonance positions of the interface. The number of spikes is determined by the relation between the thickness of the layers and the Fermi wavelength. The resonance positions of the interface are described. These positions can be split into two general classes. Some of these positions are universal and do not depend on the amplitude of the interface potential barrier and correspond to the situations when the ratio of the layer widths is given by simple rational fractions. The integer in the denominator of such fractions does not exceed the number of occupied minibands. The remaining resonance positions of the interface are nonuniversal and depend on the strength of the interface potential. In the case of a weak interface, some of these nonuniversal positions are close to the universal ones, giving the impression of a split in the conductivity spikes.

A too small width of the resonance spikes can impede the experimental observation of the geometric resonances. The width of the resonance spikes increases and their height decreases with increasing smearing of the interface and the resonance spikes gradually disappear. Note that this disappearance of the resonance spikes is related not to the widening, but to the random smearing of the interface- the widening of the interface, by itself, results just in a shift of the spike positions. The width of the universal resonances is equal, by the order of magnitude, to the width of the smeared interface $D$, Eq. (19), or, in dimensionless variables, $d$ $=D / \lambda_{F}$. The width of nonuniversal resonances is somewhat larger and is less sensitive to $D$; the reason is still unclear. Note that the smearing width $D$ can be much smaller than the physical thickness of the interface, which in metals is often larger than or of the order of the Fermi wavelength $\lambda_{F}$. In contrast to this, the smearing parameter $d=D / \lambda_{F}$ can be very small. The most stable spike with respect to smearing corresponds to the layers of equal width. The wide range of possible values of $D$, which are determined either by the roughness with $D$ of the order of $\ell$ or $\ell^{2} / R$ or by the "internal" smearing of the interface, makes the observation of the geometric resonances possible.

The spikes in the conductivity occur when the scattering probabilities for electrons in one of the quantized minibands become exactly zero. Since scattering probabilities for scat- 
tering by different interfaces add up, the spikes in conductivity of multilayer films with many layers can be observed only if the scattering probabilities for electrons from one miniband become zero simultaneously for scattering by all the interfaces. This can happen only if all the interfaces are located in the universal resonance positions corresponding to the rational fractions from the same series. Otherwise, the scattering by inhomogeneities of the "non-resonant interface" will curtail the contributions from the resonant ones. This imposes a restriction on the number of layers for an observation of this type of QSE for a film of fixed overall thickness.

We analyzed the multilayer films under the condition that the disruption in the electron spectrum be caused only by the interface potential while electron potential deep into the layers is the same for all layers. One can imagine a different physical situation when the electron potential in different layers differ from each other as in Ref. 13. In this situation the resonance spikes in conductivity should be observed when the position of the interface coincides with one of the nodes in the wave function. It is clear that this occurs at least for certain values of the interlayer potential difference $\Delta U$.

The calculations in the paper are aimed primarily at the experimental setup when the lateral conductivity is measured as a function of the film thickness at fixed thickness of the first layer (fixed position of the buried interface). The main obstacle for the experimental observation of the predicted effect is a rather small width of the conductivity spikes and their sensitivity to the position of the interface. On the other hand, this sensitivity of the QSE to the position of the interface may open the door for using this effect for precision control of the interface positions in multilayer films. This may be very useful for better quality ultrathin films without short-range surface inhomogeneities. ${ }^{18}$ Recent experiments with controlled ultrathin metal films with buried rough interfaces ${ }^{1}$ indicate that the existing experimental setups are sufficient for the observation of the predicted quantum size effect.

Usually, the QSE in the conductivity of semiconductor films is less pronounced than for metal films. This is explained by the smoother distribution of electrons in nondegenerate semiconductors. In the absence of a sharp drop in the distribution at the Fermi energy, singular features in the conductivity, which is an integral over the particle distribution, tend to be smeared out. However, the universal geometric spikes in conductivity, which are described above, are explained by the zeros in quantized electron wave functions on the interface and have nothing to do with the electron distribution. Then these spikes in conductivity can be the only striking common feature for the QSE in multilayer metal and semiconductor films. The only obstacle for the observation of such spikes in semiconductors could be a relatively large screening radius which may lead to an effective smearing of the interface.

\section{ACKNOWLEDGMENT}

This work was supported by NSF Grant No. DMR0077266 .

\section{APPENDIX: ENERGY SPECTRUM AND MATRIX ELEMENTS}

The one-dimensional Schrödinger equation for a square well with a $\delta$-functional barrier inside has the form

$$
\psi(x)^{\prime \prime}+k^{2} \psi(x)=u_{0} \delta(x-a) \psi(x),
$$

where

$$
k^{2}=2 m E / \hbar^{2}, \quad u_{0}=2 m U_{0} / \hbar^{2} .
$$

The wave functions can be written as

$$
\begin{gathered}
\psi_{1} \equiv \psi\left(x \leqslant L_{1}\right)=\sqrt{\frac{2}{L}} A \sin k x, \\
\psi_{2} \equiv \psi\left(x \geqslant L_{1}\right)=\sqrt{\frac{2}{L}} B \sin k(x-L) .
\end{gathered}
$$

In the dimensionless notation of Sec. II 2, the equation on the spectrum acquires the form

$$
\begin{gathered}
\sin (\pi z)+\frac{g}{z} \sin (\pi \delta z) \sin [\pi(1-\delta) z]=0 \\
\delta=L_{2} / L \leqslant 1, \quad k L=\pi z, \quad g=u_{0} L / \pi
\end{gathered}
$$

The normalized coefficients in the wave function (A3) are equal to

$$
\begin{gathered}
A_{n}=\frac{1}{\sqrt{\delta+(1-\delta) t_{n}^{2}+t_{n} \sin \left(z_{n} \pi\right) / z_{n} \pi}}, \\
B_{n}=A_{n} t_{n},
\end{gathered}
$$

where

$$
t_{n}=-\frac{\sin \pi \delta z_{n}}{\sin \left[\pi(1-\delta) z_{n}\right]}
$$

The explicit expression for the spectrum (A4) can be given in the limiting cases of weak and strong potential barriers. If the barrier is weak, $g / z \ll 1$, the spectrum is

$$
z_{n}=n+\Delta_{n}, \quad \Delta_{n} \approx \frac{g}{\pi n} \sin ^{2}(\pi n \delta) .
$$

In the opposite case of strong interface $g \rightarrow \infty$, the spectrum decouples into two independent series of levels for each layer:

$$
z_{n_{1}}=n_{1} / \delta, \quad z_{n_{2}}=n_{2} /(1-\delta) .
$$

For large, but finite $g$, the corrections to the spectrum (A8) can be easily obtained by expansion in $z_{n} / g \delta$ or $z_{n} / g(1$ $-\delta)$ :

$$
z_{n_{1}} \approx \frac{n_{1}}{\delta}\left(1-\frac{1}{g}\right), \quad z_{n_{2}} \approx \frac{n_{2}}{1-\delta}\left(1-\frac{1}{g}\right) .
$$


An important restriction for Eq. (A9) is that the energy levels in each of the layers, Eq. (A8), should not be very close to each other. In the case of near degeneracy, the two close levels, as usual, repel each other with a resulting gap equal to

$$
\Delta z_{n} \simeq \frac{z_{n}}{\pi g \delta(1-\delta)} .
$$

The above equations should be modified if the interface is very close to one of the external walls of the well, i.e., if either $\delta \ll 1$ or $1-\delta \ll 1$.

Note that if the $\delta$-type barrier is located exactly in the node of one the wave functions of the empty well, this wave function remains the eigenfunction of the well with a barrier inside irrespective of the strength of the barrier. This means that the energy levels that correspond to such wave functions are not shifted by the presence of the barrier.

The matrix elements of the roughness-related perturbation (6) can be calculated with the help of the above functions (A3) and (A5):

$$
\begin{aligned}
G_{n m} & =\left[\Psi_{m}\left(L_{1}\right) \Psi_{n}^{\prime}\left(L_{1}\right)+\Psi_{m}^{\prime}\left(L_{1}\right) \Psi_{n}\left(L_{1}\right)\right]^{2} \\
& =\left(\frac{2 \pi}{L^{2}} A_{m} A_{n}\right)^{2} g_{n m}^{2},
\end{aligned}
$$

$$
\begin{aligned}
g_{n m}= & g \sin \left(\pi z_{m} \delta\right) \sin \left(\pi z_{n} \delta\right)+z_{m} \cos \left(\pi z_{m} \delta\right) \sin \left(\pi z_{n} \delta\right) \\
& +z_{n} \cos \left(\pi z_{n} \delta\right) \sin \left(\pi z_{m} \delta\right) .
\end{aligned}
$$

The most important are the diagonal matrix elements

$$
\begin{aligned}
g_{n n} & =\sin \left(\pi z_{n} \delta\right)\left[g \sin \left(\pi z_{n} \delta\right)+2 z_{n} \cos \left(\pi z_{n} \delta\right)\right] \\
& =\frac{z_{n} \sin \left(\pi z_{n} \delta\right) \sin \left[\pi z_{n}(1-2 \delta)\right]}{\sin \left[\pi z_{n}(1-\delta)\right]} .
\end{aligned}
$$

Note that the zeros of the denominator in Eq. (A12) are canceled out by the zeros of $A_{n}^{2}$, Eq. (A11). When the interface has a finite width $d$, the matrix elements acquire the following addition:

$$
g_{n m}^{2(t o t)}=g_{n m}^{2}+d^{2}\left(\Delta g_{n m}\right)^{2},
$$

where

$$
\begin{aligned}
\Delta g_{n m}= & 2 z_{n} z_{m} \cos \left(\pi z_{n} \delta\right) \cos \left(\pi z_{m} \delta\right) \\
& -\left(z_{n}^{2}+z_{m}^{2}\right) \sin \left(\pi z_{n} \delta\right) \sin \left(\pi z_{m} \delta\right)+g g_{n m} .
\end{aligned}
$$

The total matrix element $G_{n m}^{(t o t)}$ is never zero. This means that the term with $d^{2}$, which originates from the smearing of the interface, provides a natural cutoff for the conductivity.

In degenerate metal films, of all the energy minibands $\epsilon_{n}$, only the minibands with $n \leqslant \operatorname{Int}\left[L / \lambda_{F}\right]$ are occupied.
${ }^{1}$ I. B. Altfeder, D. M. Chen, and K. A. Matveev, Phys. Rev. Lett. 80, 4895 (1998); I. B. Altfeder, V. Narayanamurti, and D. M. Chen, ibid. 88, 206801 (2002).

${ }^{2}$ A. E. Meyerovich and I. V. Ponomarev, Phys. Rev. B 65, 155413 (2002).

${ }^{3}$ P. M. Levy, in Solid State Physics, edited by H. Ehrenreich and D. Turnbull (Academic, New York, 1994), Vol. 47, pp. 367-462.

${ }^{4}$ P. Joyez and D. Esteve, Phys. Rev. B 64, 155402 (2001); S. V. Korepov and M. A. Liberman, ibid. 60, 13770 (1999); J. Barnas and G. Palasatzas, J. Appl. Phys. 82, 3950 (1997).

${ }^{5}$ M. Jalochowski, M. Hoffmann, and E. Bauer, Phys. Rev. Lett. 76, 4227 (1996); Phys. Rev. B 51, 7231 (1995); L. A. Kuzik, Yu. E. Petrov, F. A. Pudonin, and V. A. Yakovlev, Sov. Phys. JETP 78, 114 (1994); G. M. Mikhailov, I. V. Malikov, and A. V. Chernykh, JETP Lett. 66, 725 (1997).

${ }^{6}$ M. Jalochowski, E. Bauer, H. Knoppe, and G. Lilienkamp, Phys. Rev. B 45, 13607 (1992); M. Jalochowski, M. Hoffmann, and E. Bauer, ibid. 51, 7231 (1995); H. Sakaki, T. Noda, K. Hirakawa, M. Tanaka, and T. Matsusue, Appl. Phys. Lett. 51, 1934 (1987); L.-W. Tu, G. K. Wong, and J. B. Ketterson, ibid. 55, 1327 (1989).

${ }^{7}$ S. Andrieu, C. Chatelain, M. Lemine, B. Berche, and Ph. Bauer, Phys. Rev. Lett. 86, 3883 (2001).

${ }^{8}$ J. J. Paggel, T. Miller, and T. C. Chang, Science 283, 1709 (1999); D. A. Evans, M. Alonso, R. Cimino, and K. Horn, Phys.
Rev. Lett. 70, 3483 (1993); J. E. Ortega, F. J. Himpsel, G. J. Mankey, and R. F. Willis, Phys. Rev. B 47, 1540 (1993).

${ }^{9}$ A. E. Meyerovich and S. Stepaniants, J. Phys.: Condens. Matter 9, 4157 (1997).

${ }^{10}$ V. B. Sandomirskii, Zh. Éksp. Teor. Fiz. 52, 158 (1968) [Sov. Phys. JETP 25, 101 (1967)].

${ }^{11}$ N. Trivedi and N. W. Ashcroft, Phys. Rev. B 38, 12298 (1988).

${ }^{12}$ G. Palasantzas, Y.-P. Zhao, G.-C. Wang, T.-M. Lu, J. Barnas, and J. Th. M. De Hosson, Phys. Rev. B 61, 11109 (2000); G. Palasantzas and J. Th. M. De Hosson, ibid. 63, 125404 (2001).

${ }^{13}$ A. E. Meyerovich and A. Stepaniants, Phys. Rev. B 60, 9129 (1999).

${ }^{14}$ Yu. I. Latyshev, A. A. Sinchenko, L. N. Bulaevskii, V. N. Pavlenko, and P. Monceau, JETP Lett. 75, 93 (2002).

${ }^{15}$ G. Fishman and D. Calecki, Phys. Rev. Lett. 62, 1302 (1989).

${ }^{16}$ Z. Tesanovic, M. V. Jaric, and S. Maekawa, Phys. Rev. Lett. 57, 2760 (1986); A. E. Meyerovich and S. Stepaniants, Phys. Rev. Lett. 73, 316 (1994).

${ }^{17}$ A. E. Meyerovich and A. Stepaniants, J. Phys.: Condens. Matter 12, 5575 (2000).

${ }^{18}$ J. J. Paggel, T. Miller, and T. C. Chiang, Phys. Rev. Lett. 81, 5632 (1998); Science 283, 1709 (1999); F. Patthey and W.-D. Schneider, Phys. Rev. B 50, 17560 (1994); M. Schmid, W. Hebenstreit, P. Varga, and S. Crampin, Phys. Rev. Lett. 76, 2298 (1996). 